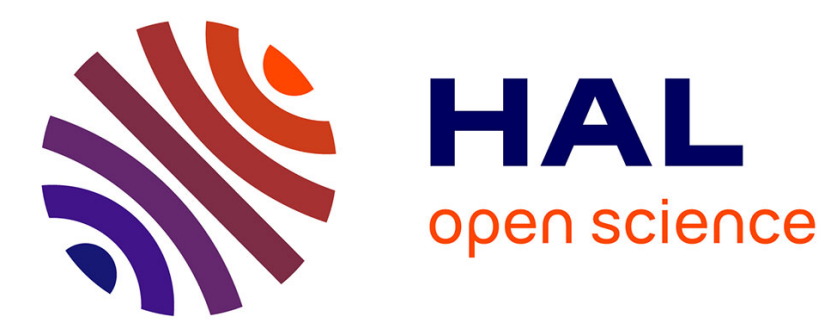

\title{
3D Interaction Assistance Through Context-Awareness
}

Yannick Dennemont, Guillaume Bouyer, Samir Otmane, Malik Mallem

\section{To cite this version:}

Yannick Dennemont, Guillaume Bouyer, Samir Otmane, Malik Mallem. 3D Interaction Assistance Through Context-Awareness. Virtual Reality (VR 2012), Mar 2012, Orange County, CA, United States. pp.103-104, 10.1109/VR.2012.6180903 . hal-00665627

\section{HAL Id: hal-00665627 https://hal.science/hal-00665627}

Submitted on 2 Feb 2012

HAL is a multi-disciplinary open access archive for the deposit and dissemination of scientific research documents, whether they are published or not. The documents may come from teaching and research institutions in France or abroad, or from public or private research centers.
L'archive ouverte pluridisciplinaire HAL, est destinée au dépôt et à la diffusion de documents scientifiques de niveau recherche, publiés ou non, émanant des établissements d'enseignement et de recherche français ou étrangers, des laboratoires publics ou privés. 


\title{
3D Interaction Assistance Through Context-Awareness
}

\author{
Yannick Dennemont Guillaume Bouyer Samir Otmane Malik Mallem
}

IBISC Laboratory, Evry University, France.*

\begin{abstract}
This work focuses on enabling 3D interaction assistance by adding adaptivity depending on the tasks, objectives, and the general interaction context. We model the context using Conceptual Graphs (CG) based on an ontology. Including CG in our scene manager (Virtools) allows us to add semantic information and to describe the available tools. We handle rules leading to adaptation with a logic programming layer (Prolog+CG) included in the Amine platform. This project is a step towards Intelligent Virtual Environments, which proposes a hybrid solution by adding a separate semantic reasoning to classic environments. The first case study automatically manages few modalities depending on the distance to objects, user movement, available tools and modality risks.
\end{abstract}

Index Terms: I.3.6 [Methodology and Techniques Subjects]: Interaction Techniques- [I.3.7]: Computer Graphics-Virtual reality I.2.4 [Computing Methodologies]: Knowledge Representation Formalism and Methods-

\section{INTRODUCTION AND PROBLEMATICS}

3D interaction (3DI) immersion and usability are reinforced by the use of natural schemes. Meantime, adaptivity based on context is natural in human communication. Thus, we are designing a context and decision manager that focuses on expressiveness and usability in order to add adaptivity to 3DI. Through exploiting the context, we plan to best determine means to effectively help the user.

3DI adaptation is an approach suggested by many researches [3] [7], where some of its advantages are: speeding up the interaction, diminishing the cognitive load, tailoring the interaction[7] and adding or managing interaction possibilities [2]. As the "perfect" 3DI technique has yet to be found, a solution can be to adapt the interaction to a task, a device, etc., by adding specificity. Flavors, i.e known technique variations, to improve usability are another form of adaptation. Applying automatically these types of adaptations when needed defines an adaptative 3DI, which can be implicit with adaptations embedded in the interaction techniques, or explicit by using external processes [2][7]. Adaptive systems can provide a recognition of higher level information from raw data (gestures, tasks, etc.). However, to achieve a better adaptivity, we need more content description : the context.

A formal and well recognized definition of context is [5]: Context is any information that can be used to characterize the situation of an entity. An entity is a person, place, or object that is considered relevant to the interaction between a user and an application, including the user and applications themselves.

Thus, our project fits the definition of context-awareness (CA) as we use context to provide relevant information and/or services to the user, where relevancy depends on the user's task [5].

The CA forms share an ideal list of properties to handle [1]:

- Heterogeneity and mobility of context.

- Relationships and dependencies between context.

*e-mail: firstname.lastname@ibisc.univ-evry.fr
- Timeliness: access to previous and future states.

- Imperfection: data can be uncertain and even incorrect.

- Reasoning: decide or derive higher level context.

- Usability of modelling formalisms.

- Efficient context provisioning.

\section{KnOWledge Representation and ReAsoning}

We need to manage context and decide how to react, which is a form of Knowledge Representation and Reasoning (KR \& R). Both parts are not totally independent and expressive representations are usually less reasoning efficient. Representations are various (keyvalues, markup-models, ontologies, graphical models, etc.). For reasoning, we can use bi-valued logic (description logic, first order logic, higher order logic), multi-valued logic or other forms of multi-valued models (fuzzy logic, bayesian networks, hidden Markov model, neural networks) that rely on different semantics.

Several criteria have motivated our choice: semantic degrees, expressiveness (vs efficiency) and usability. We chose to base our representation on Conceptual Graphs (CG) that have a strong semantic founding and are built on an ontology. Furthermore, they provide a good expressiveness (a universal knowledge representation) equivalent to First Order Logic (FOL), but with a better usability since they are human readable as well. The needed expressiveness is an open issue, but also a fundamental question for a sustainable use. FOL is usually the most expressive choice made for CA. Moreover, coupled with the CG usability and its ontology, the model is not fully abstract and we may allow, at some point, a direct involvement of users [4] . In fact, semantic virtual worlds as a new paradigm is already a discussed issue. However, we will not try to build a full semantic world but rather to gather semantic information in order to help the 3DI. Our goal is to obtain CA in none-semantic applications with an external representation and reasoning engine.

\section{CurRent AND FUtURE WORK}

As shown in Figure 1, the engine manages context and decisions concerning the user, interaction and environment, which communicate through different tools. Those tools must have a semantic description of their uses to be triggered by the engine. They can be actuators with visible effects or sensors that retrieve information.

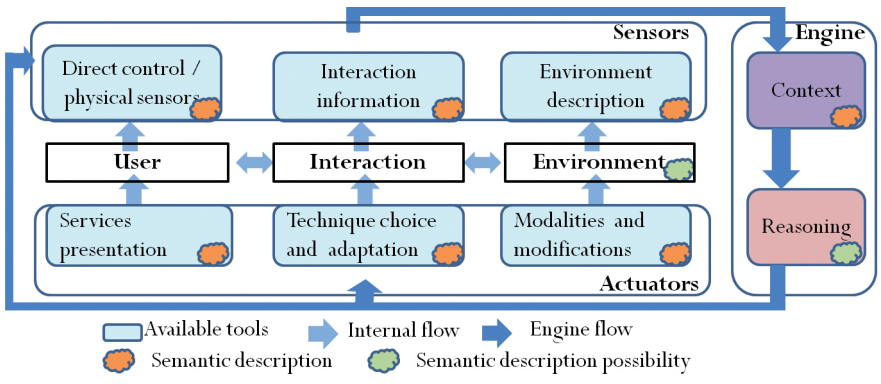

Figure 1: A Parallel Engine - communication though semantic tools

Context can have various forms as illustrated in Figure 2. First, the ontology lists concepts and relations with underlying semantic, which are used by CG in order to describe rules and facts. Available tools and the past events in history are special facts. Events are 
newly integrated information and trigger a decision request in an automatic mode. The time manager role is to check the validity of the needed facts. When a decision with an associated tool is true, the engine aggregates its belief and risk from facts, events' timing and rules. The user cognitive load is represented by an acceptable total risk, which induces a knapsack problem as a last classification.

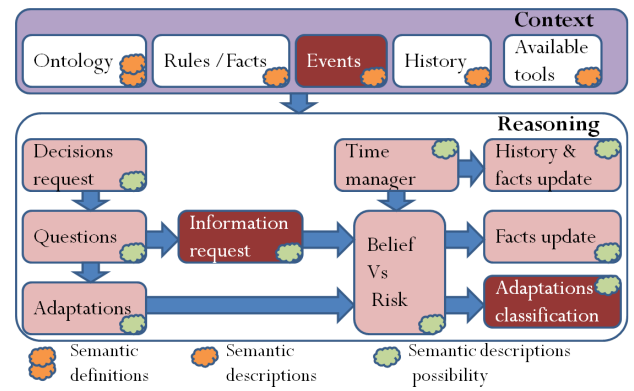

Figure 2: The Engine - forms of context and reasoning

Context and reasoning blocks (as shown in Figure 2) can also be grouped by their role in the engine as shown in Figure 3. The situation progresses with two roughly separated interlaced processes : decision and comprehension. The decision process goes from representing the situation to reasoning ( $K R \& R$ ), and transforming simple facts beliefs into the best reaction bet. The comprehension process goes from identifying the situation to understanding how to assist (the two CA trends) and transform simple data to a full plan of the situation. The decision set is extended as the comprehension progresses (e.g an acquired interest can unlock a reactive adaptation)

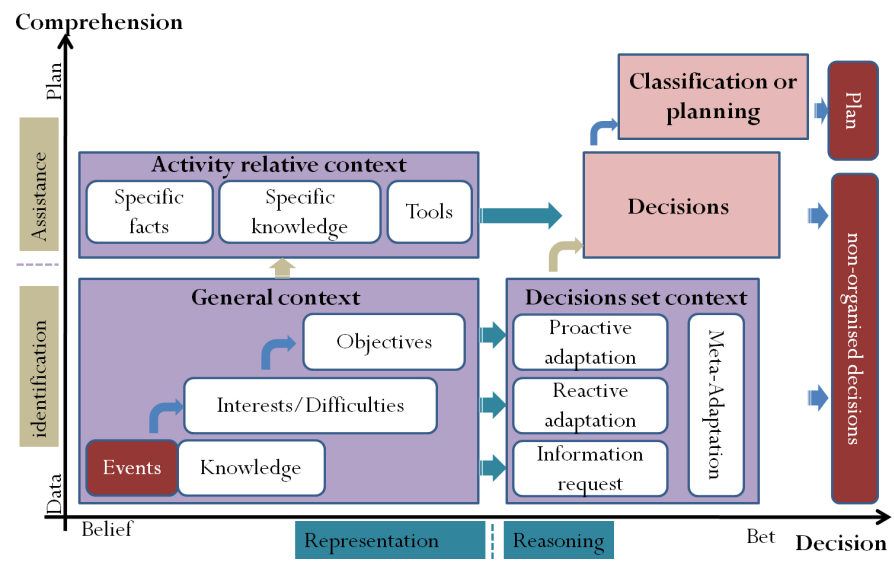

Figure 3: Hierarchy in the engine - Towards an assistance plan bet

We use Virtools as our scene graph manager and the Amine platform [6] (a Java open-source multi-layer platform for intelligent systems) for the engine. This platform offers an ontology manager and a FOL backward chaining engine that handles CG :Prolog+CG.

We are testing the engine on a small scenario. However, even simple cases pose several open questions: What items of information are pertinent? How to balance decisions risk and belief? How to best express a rule? How to monitor the user's intention?.

The engine core combines general rules with scene tools and specific rules. We test the engine and those rules with a first case study: to try to automatically acquire some user's interests and enhance them. We first focus on interests linked to the user's hand. Thus the only specific rule is to monitor the "hand" object and to set a cognitive load. Then, several tools may help. We started by defining:

- a Zones Of Interest (ZOI) sensor that attaches auras to objects and report their content

- a object's movement sensor (movement is high/low, local/global)

- an actuator to change the color of an object
- an actuator to add a haptic or visual gain to an object

The engine's first reaction is to activate the sensors on the target "hand". Those sensors send events to the engine, e.g an event that describes an object inside the hand's ZOI. Events trigger a reaction request that finds adaptations with corresponding available tools: e.g both precedent actuators, with two uses for activation and deactivation. Some of the needed general rules are :

- Define interests (e.g in a ZOI)

- Try to enhance an interest.

- Associate possible enhancements in this situation : e.g object visual modifications through color change, as well as interaction modifications through gain (visual or haptic).

- Manage adaptations states (possibly with previous decisions)

- remove added visual modifications if object is not an interest.

- remove a gain if an object is an interest and the movement is abnormal (e.g local+high=the user is "stuck").

Next, the engine calculates the decisions'belief and risk from the initial risk supplied by the tools and possibly beliefs for sensors events. Each rule has a belief transfer rate. This rate for a color change is greater than the one for a visual gain, as it is usually more suited with only the "enhancement will" context. Finally, we add rules to manage risk:

- increase decision risk if some concepts are used (e.g haptic gain risk $>$ visual gain risk $>$ object visual modification risk)

- increase decision's risk if present in history (e.g avoid activation/deactivation cycle)

- decrease interaction modification's risk if movement is local As a result, the rules combine themselves as expected. Passing by an object makes its color red, while standing next to it makes it also attractive. Colors are reset when we move far away, while attraction is removed when we try to resist it. When it has been deactivated, gain cannot be reactivated for a time corresponding to history memory. Some reactivations can occur for coloring as the decision is initially less risky. Those results depend on the initial risk, belief and cognitive load values. More complex situations occur when several objects are close to the hand: e.g only the less risky adaptation is applied to a maximum of objects.

The setting up and implementation of an intelligent 3D interaction assistance and context manager with Amine platform and Conceptual Graphs is promising, and we are now improving it by reflections on examples. The engine aims to allow a semantic reasoning and the reuse of tools in a non-semantic environment to help the $3 \mathrm{D}$ interaction. We propose an engine core with a semantic base to achieve adaptation, which could be directly addressed by sensors, designers or users. Context and adaptations to be considered for virtual reality along with the user's degrees of appreciation and control are open questions which will guide the engine evolution. The next major step is to obtain enhanced user's intention hints.

\section{REFERENCES}

[1] C. Bettini, O. Brdiczka, K. Henricksen, J. Indulska, D. Nicklas, A. Ranganathan, and D. Riboni. Pervasive and Mobile Computing, 2010.

[2] G. Bouyer, P. Bourdot, and M. Ammi. Supervision of Task-Oriented Multimodal Rendering for VR Applications. Communications, 2007.

[3] D. A. Bowman, J. Chen, C. A. Wingrave, J. Lucas, A. Ray, N. F. Polys, Q. Li, Y. Haciahmetoglu, J.-s. Kim, S. Kim, R. Boehringer, and T. Ni. New Directions in 3D User Interfaces. International Journal, 5, 2006.

[4] P. Brézillon. From expert systems to context-based intelligent assistant systems : a testimony. Engineering, 26:19-24, 2011.

[5] A. Dey and G. Abowd. Towards a better understanding of context and context-awareness. In CHI 2000 workshop on the what, who, where, when, and how of context-awareness, volume 4, 2000.

[6] A. Kabbaj. Development of Intelligent Systems and Multi-Agents Systems with Amine Platform. Intelligence, pages 1-14, 2006.

[7] J. Octavia, K. Coninx, and C. Raymaekers. Enhancing User Interaction in Virtual Environments through Adaptive Personalized 3D Interaction Techniques. In UMAP, 2010. 